on intrathoracic surgery has been modernized, and diagrams of endobronchial intubation have been added. The operation for coarctation of the aorta receives brief mention, but anaesthesia for Blalock's operation, which bristles with interesting problems, is not discussed.

Weights and measures are given arbitrarily in English or in metric units. It is a pity that the chance has been lost in this new edition, which will be so widely read, of striking a blow for uniformity by expressing them all in the metric system, with the English equivalents, when these are more familiar, in parentheses.

The book is excellently produced, and Messrs. Livingstone are to be congratulated for surpassing their already high standard. Many new illustrations, some in colour, have been added, and much enhance its value. The touched up photograph of McMurray's intra-nasal administrator in position on what appears to be a dissecting room specimen is one of the few which might, with advantage, be omitted from subsequent editions. Apart from such very minor blemishes, this book is a model of what a textbook should be. It is well balanced, embracing in scope, and full of knowledge and practical instruction, presented in a lucid style which makes it. a delight to read. Its price has gone up by twenty per cent. : its worth by more than that.

$$
\text { R.F.W. }
$$

\section{ORAL AND DENTAL DISEASES}

By Hubert H. Stones, M.D., M.D.S., F.D.S., R.C.S., (Eng.) Prof. of Dental Surgery and Director of Dental Education, University of Liverpool. Hon. Director Liverpool Dental Hospital, etc. Edinburgh, E. and S. Livingstone, Ltd. 1948. Pp. 896. 926 illustrations, 82 in colour. 9os.

The need for a book such as this has long been felt and Prof. Stones should be congratulated on its production.

It deals mainly with the aetiology, pathology and diagnosis of oral and dental diseases and while general treatment for various conditions is outlined, specialized surgical treatment is, on the whole, only briefly described. The exposition is clear, the subject matter well up to date and the illustrations, some of which are in colour, are excellent.

There is a useful list of references at the end of each chapter so that further information on any subject is easily obtained.

This book. should prove of great value, both to students and to practitioners and the judgements given, for example, in the section on the relationship of focal infection and the teeth, are invariably sound. It is a pleasure to read a book in which so little modern work appears to have been omitted and yet in which the whole field is adequately covered in a clear and lucid style.

This book can be recommended with confidence.

\section{A.B.M.}

\section{IDENTIFICATION OF TUMOURS}

Essential Gross and Microscopic Pathologic Features Systematically Arranged for EAsier IDENTIFICATION

By N. Chandler Foot, M.D., Professor of Surgical Pathology, Cornell University Medical College; Surgical Pathologist to New York Hospital. J. B. Lippincott Company. 1948. . Pp. 397. 24I illustrations. 36s:

Professor Foot has certainly succeeded in fulfilling his objective given in the title above. Any doubts as to the place for such a book are dispelled at once on reference to this lucid and balanced work which, by assuming a moderate preliminary knowledge of pathology on the reader's part, gets straight down to essential points in the diagnosis of tumours.

Part I, 96 pages, covers neoplasms of general distribution in an orthodox manner pointing out the source, site, age and sex, gross appearance, microscopic appearance, differential diagnosis, metastatic properties, prognosis and analogues of growths of fibrous tissue, cartilage and bone, muscle, fat, serous membranes, cardiovascular system, epithelial tissue, lymphoid tissue and spleen. Part II, 254 pages, deals similarly with tumours peculiar to specific systems and organs; respiratory system and mediastinum, alimentary system, liver, gall bladder and pancreas, urinary system, organs of internai secretion, male reproductive system, female reproductive system, breast, nervous system, skin, ear and eye. Space and repetition are saved by references back to Part I. There follows a chapter on methods of fixation and staining and then for 35 pages a ' tabular locator for tentative identification of neoplasms.' 'This is really a condensation of Parts I and II, the tumours are arranged in the same order and their appearances and properties tabulated under the headings morphology, type cell, stroma or matrix, differentiation, mitotic figures, other features, diagnosis. Finally there is an index.

The book is well arranged, beautifully produced and easy to use. The 24I illustrations, all photomicrographs in black and white, are of good but by no mears superlative standard. They must be studied together with the text description for full benefit; the magnifications are well chosen. It is a comprehensive work and describes the rarer neoplasms as well as infrequent varients of the common ones.

The text is full of interesting points by which the author passes on the results of his extensive experience to the less experienced observer. Examples are :- the presence of elastic fibres in soft fibromas, the brownish colour of fibrosarcomas, the usefulness of Masson's stain to pick out nerve fibres; the description of a massive thoracic tumour derived from intercostal fascia, the bringing out of myxoma cell stellate processes by silver impregnation, the presence of osteoclasts in myositis ossificans, the absence of reticulin fibres in Ewing's bone sarcoma, the mimicking of rhabdomyosarcoma by some types 